\title{
Governamentalidade algorítmica e a rarefação dos processos de subjetivação
}

\author{
Algorithmic governmentality and the rarefaction of subjetivaction \\ processes
}

Guilherme Primo ${ }^{1}$

\section{Resumo}

Este trabalho tem por escopo examinar os conceitos foucaultianos de governamentalidade e dispositivos de segurança, de modo a retomá-los numa abordagem contemporânea, que leve em conta a análise dos dispositivos algorítmicos e seus efeitos de saber diante das relações virtuais estabelecidas com as novas tecnologias digitais. Para avançar na questão, utilizarei do conceito de governamentalidade algorítmica, elaborado por Antoinette Rouvroy e Thomas Berns, de modo a apreender o movimento através do qual esses mecanismos operam sobre as interações virtuais, estabelecendo correlações entre dados e elaborando perfis de comportamento, a partir dos quais define um conjunto de estratégias e antecipações sobre ações possíveis, neutralizando as subjetividades e dispensando o mundo e suas representações, em nome de uma realidade feita de fluxos e sinais numéricos.

Palavras-chave: Michel Foucault. Governamentalidade. Algoritmos. Biopolítica.

\section{Abstract}

The scope of this work is to examine Foucault's concepts of governmentality and security dispositifs, in order to retake them in a contemporary approach, which takes into account the analysis of algorithmics dispositifs and their effects of knowledge in face of virtual relationships established with the new digital technologies. To enter in the question, I will use the concept of algorithmic governmentality, developed by Antoinette Rouvroy and Thomas Berns, in order to understand the movement through which these mechanisms operate on virtual interactions, establishing correlations between data and developing behavior profiles, and defining a set of strategies and anticipations about possible actions, neutralizing subjectivities and dispensing the world and it's representations, in the name of a reality made up of flows and numerical signs.

Keywords: Michel Foucault. Governmentality. Algorithms. Biopolitics.

\footnotetext{
${ }^{1}$ Doutorando pelo Programa de Pós-Graduação em Filosofia da PUCRS, com bolsa fomentada pela CAPES.

E-mail: guiprimo_@hotmail.com
} 


\section{Introdução}

Este trabalho se divide em duas partes. Num primeiro momento, retomaremos os conceitos foucaultianos de dispositivos de segurança e governamentalidade, ainda que brevemente, de modo a realizar, a partir daí, uma abordagem contemporânea com o objetivo de analisar os dispositivos algorítmicos e seus efeitos de saber diante das relações virtuais estabelecidas com as novas tecnologias digitais. Em seguida, avançaremos na questão, tomando por base o conceito de governamentalidade algorítmica, desenvolvido por Antoinette Rouvroy e Thomas Berns, procurando apreender os movimentos através dos quais tais dispositivos operam, de modo a estabelecer correlações entre dados coletados a partir da interatividade virtual e elaborando perfis de comportamento.

Conforme veremos, a exumação das estruturas que compõem esse novo campo de saber, que emerge do ambiente digital contemporâneo, bem como das novas maneiras de relacionar-se, parece-nos imprescindível, na medida em que tais mecanismos não apenas definem um conjunto de estratégias e antecipações sobre ações possíveis, mas agem de modo a neutralizar as subjetividades e o mundo, intervindo sobre as relações em nome de uma realidade composta por fluxos e sinais numéricos - e, sobretudo, levando a uma radicalização das opiniões, bem como a um desaparecimento da experiência comum e encerramento das possibilidades de agir político.

\section{O conceito de governamentalidade em Michel Foucault}

Para que possamos adentrar a questão, faz-se necessário retomarmos alguns conceitos fundamentais das obras de Michel Foucault, especialmente aqueles que remetem à segunda metade dos anos 70, momento em que seus escritos inclinamse em direção a uma genealogia dos dispositivos de segurança, bem como à formação do conceito de governamentalidade.

Com a publicação do primeiro volume de Histoire de la sexualité, I: la volonté de savoir (1976), a análise das relações de poder adquire uma nova acentuação, na medida em que Foucault descreve a formação do discurso sobre o sexo como o ponto de entrecruzamento entre os dois eixos do biopoder. Pois, conforme Foucault, 
Sur ce fond, peut se comprendre l'importance prise par le sexe comme enjeu politique. C'est qu'il est à la charnière des deux axes le lon desquels s'est développée toute la technologie politique de la vie. D'un côté il relève des disciplines du corps: dressage, intensification et distribution des forces, ajustement et économie des énergies. De l'autre, il rèleve de la regulation des populations, par tous les effets globaux qu'il induit ${ }^{2}$.

Se, com o exame dos dispositivos disciplinares, em Surveiller et punir (1975), Foucault colocou em cheque a centralidade da concepção jurídica de poder, escorada sobre categorias modernas como lei e soberania, em La volonté de savoir sua análise avança, de modo a compreender a hipótese repressiva sobre a sexualidade dentro de uma economia geral dos discursos sobre o sexo, que se desenvolveram no seio da Modernidade, especialmente desde o século XVII.

Diante da genealogia do dispositivo da sexualidade, Foucault identifica a formação de um saber que passa a levar em conta não apenas a anátomo-política do corpo humano (anatomo-politique du corps humain) ${ }^{3}$, como no caso das disciplinas, mas, fundamentalmente, uma biopolítica da população (bio-politique de la population $)^{4}$, centrada no emergir do corpo-espécie enquanto objeto e objetivo de uma série de intervenções e controles. Diferentemente do poder soberano, que marcava a vida através de uma exigência de morte, o emergir da Modernidade trouxe consigo uma profunda transformação nos mecanismos do poder, destarte orientados ao gerenciamento da vida, de modo a majorá-la, multiplicá-la, e exercer sobre ela controles e regulações de conjunto. "On pourrait dire qu'au vieux droit de faire mourir ou de laisser vivre s'est substitué un pouvoir de faire vivre ou de rejeter dans la mort" 5

Conforme Foucault, o emergir dessa tecnologia de duas faces - disciplinar e biopolítica - foi elemento indispensável para o desenvolvimento do capitalismo, cujo funcionamento pôde ser garantido mediante "l'insertion contrôlée des corps dans l'appareil de production et moyennant un ajustement des phénomènes de population aux processus économiques" ${ }^{6}$. Não se está a tratar, portanto, da Modernidade enquanto aurora de uma compaixão humanitária e universal, mas do ponto a partir do qual o homem ocidental coloca a vida no centro dos cálculos

\footnotetext{
${ }^{2}$ FOUCAULT, Michel. Histoire de la sexualité, I: la volonté de savoir. Paris: Éditions Gallimard, 1976, p. 191-192.

3 Ibidem, p. 183.

4 Ibidem, p. 183.

5 Ibidem, p. 181.

6 Ibidem, p. 185. 
políticos, através da abertura de uma nova dimensão de saber.

\begin{abstract}
Pour la première fois sans doute dans l'histoire, le biologique se réflechit dans le politique; le fait de vivre n'est plus ce soubassement inaccessible qui n'émerge que de temps en temps, dans le hasard de la mort et sa fatalité; il passe pour une part dans le champ de contrôle du savoir et d'intervention du pouvoir. Celui-ci n'aura plus affaire seulement à des sujets de droit sur lesquels la prise ultime est la mort, mais à des êtres vivants, et la prise qu'il pourra exercer sur eux decra se placer au niveau de la vie elle-même; c'est la prise en charge de la vie, plus que la menace du meutre, qui donne au pouvoir son accès jusqu'au corps. Si on peut appeler 'bio-histoire-' les pressions par lesquelles les mouvements de la vie et les processus de l'histoire interfèrent les uns avec les autres, il faudrait parler de 'biopolitique' pour désigner ce qui fait entrer la vie et ses mécanismes dans le domaine des calculs explicites et fait du pouvoir-savoir un agent de ttransformation de la vie humaine $[. .$.$] .$
\end{abstract}

La volonté de savoir demarca, portanto, um ponto de inflexão nas obras de Foucault, articulando a relação entre as disciplinas e a biopolítica, e apontando o novo horizonte das investigações do autor. Em 1978, em seu curso Securité, territoire, population, ministrado no Collège de France, Foucault aprofunda suas análises, oferecendo uma nova leitura sobre as relações históricas entre os diversos dispositivos de poder-saber, e introduz a categoria dos dispositivos de segurança (dispositifs de sécurité) em relação à biopolítica.

A genealogia desses dispositivos remete a vários pontos de emergência, explorados pelo autor - especialmente a partir do século XVI, como nas discussões sobre a organização do espaço urbano na França; diante do fenômeno de escassez alimentar dos séculos XVII e XVIII na Europa; mesmo acerca das epidemias, como da varíola, e as campanhas de inoculação do século XVIII -, no contexto de outros mecanismos, como o poder soberano e as estruturas disciplinares. Ressaltemos, entretanto, que Foucault deixa claro não afirmar uma linearidade histórica, ou uma série na qual os elementos vão se suceder, entre o poder jurídico-legal, as disciplinas e os dispositivos de segurança. De fato, trata-se de uma "une série d'édifices complexes dans lesquels ce qui va changer, bien sûr, ce sont les techniques ellesmêmes qui vont se perfectionner, ou en tout cas se compliquer, mais surtout ce qui va changer, c'est la dominante ou plus exactement le système de corrélation entre les mécanismes juridico-legaux, les mécanismes disciplinaires et les mécanismes de sécurité"8.

7 Ibidem, p. 187-188.

${ }^{8}$ FOUCAULT, Michel. Securité, territoir, population. Cours au Collège de France (1977-1978). Paris: Seuil/Gallimard, 2004, p. 10. 
O que se pode entender, então, por "segurança", no sentido emprestado ao termo por Foucault? De maneira geral, os dispositivos de segurança são um conjunto de mecanismos e táticas, em cujo escopo está a inserção de um fenômeno numa série de acontecimentos prováveis, elaborando suas reações ante tal fenômeno a partir de um cálculo de custo, e estabelecendo os limites entre o aceitável e o não aceitável através da fixação de uma média considerada ótima.

Os dispositivos de segurança designam um sistema centrado na aleatoriedade desses acontecimentos e fenômenos eventuais, como a escassez alimentar e as epidemias, buscando impedi-los ou programá-los antes de sua inscrição na realidade. Ele se constitui a partir do que já está dado, ou seja, minimizando os riscos e os inconvenientes, e maximizando os elementos positivos, abrindo-se ao futuro e ao que pode acontecer. "C'est la gestion de ces séries ouvertes, et par conséquent qui ne peuvent être contrôlées que par une estimation de probabilités, c'est cela, je crois, qui caractérise assez essentiellement le mécanisme de sécurité" 9 . Trata-se de intervir no suporte através do qual essas ações circulam, ou seja, no espaço em que a série de acontecimentos aleatórios se desenrolam, que, conforme Foucault, poderia ser denominado meio (milieu) ${ }^{10}$, tomando-se o significado emprestado ao termo pela biologia ou pela física: o meio designa aquilo que é necessário para explicar a ação à distância de um corpo sobre outro ${ }^{11}$.

A noção de meio, portanto, adquire relevância fundamental na formação dessas estratégias de poder-saber, levando Foucault a realizar uma distinção necessária entre as noções de normação (normation) e normalização (normalisation) ${ }^{12}$. Pois, na medida em que os mecanismos de segurança distanciam-se do fenômeno para apreender o ponto em que as coisas vão se produzir, operando uma regulação no elemento da realidade, já não estamos diante de uma força centrípeta, como no poder disciplinar, cujo primeiro gesto está na circunscrição do espaço no qual seus mecanismos funcionarão plenamente. A disciplina se funda no caráter primitivamente descritivo da norma, definindo aquilo que é considerado normal ou anormal, construindo um modelo em relação ao qual se espera certo resultado, e seu objetivo consiste em tornar pessoas, atos e gestos conformes a esse modelo.

\footnotetext{
9 Ibidem, p. 22

10 Ibidem, p. 22.

${ }^{11}$ Ibidem, p. 22.

12 Ibidem, p. 59.
} 
Deste modo, Foucault prefere atribuir, a propósito das técnicas disciplinares, o conceito de normação, visto que apenas em relação à norma era possível distinguir a normalidade ou a anormalidade. Os dispositivos de segurança, por outro lado, não descrevem uma norma preambular, mas operam uma identificação do normal e do anormal, das diferentes curvas de normalidade, com o objetivo de fazer com que essas diferentes distribuições de normalidade funcionem umas em relação às outras. Enquanto nas disciplinas a norma é anterior e externa, no caso dos dispositivos de segurança, pelo contrário, ela é intrínseca, seguindo a tendência geral que os acontecimentos descrevem: portanto, não mais uma normação, mas uma normalização. Nesse sentido, conforme Foucault,

On a donc là quelque chose qui part du normal et qui se sert de certaines distributions considérées, si vous voulez, comme plus normales que les autres, plus favorables en tout cas que les autres. Ce sont ces distributionslà qui vont servir de norme. La norme est un jeu à l'intérieur des normalités différientieles. C'est le normal qui est premier et c'est la norme qui s'en déduit, ou c'est à partir de cette étude des normalités que la norme se fixe et joue son rôle opératoire. Donc, je dirais là qu'il ne s'agit plus d'une normation, mais plutôt, au sens strict enfin, d'une normalisation ${ }^{13}$.

A figura da população, que, conforme vimos, já havia sido introduzida por Foucault em La volonté de savoir, assume um papel elementar na conformação dos dispositivos de segurança, junto às noções de meio, aleatoriedade e normalização, tendo em vista que, no escopo desses mecanismos, não está em jogo a multiplicidade dos sujeitos jurídicos, como no caso da soberania, ou a multiplicidade dos corpos individuais, objeto dos dispositivos disciplinares: seu objetivo está em atingir uma população, “une multiplicité d'individus qui sont et qui n'existent que profondément, essentiellement, biologiquement liés à la materialité à l'intérieur de laquelle ils existent" ${ }^{14}$.

Procura-se intervir e regular a realidade da população, visando estabelecer uma relação econômica com o fenômeno da circulação das causas e dos efeitos, que sucede dentre os dados naturais de um determinado meio. "Ce qu'on va essayer d'atteindre, par ce milieu, c'est là où précisément interfère une série d'événements que ces individus, populations et groupes produisent, avec des événements de type quasi naturel qui se produisent autour d'eux" ${ }^{5}$.

\footnotetext{
13 Ibidem, p. 65.

14 Ibidem, pg. 23.

15 Ibidem, pg. 23.

Opinião Filosófica - ISSN: 2178-1176 - Editora Fundação Fênix. www.fundarfenix.com.br
} 
A emergência do problema da população e o isolamento desse nível de realidade, através da estatística, trouxe consigo, portanto, o desbloqueio da arte de governar (l'art de gouverner), possibilitando o recentrar da noção de economia para além da família, e introduzindo-a, deste modo, no seio do exercício político. E, nesse sentido, governar significa encarregar-se dos homens em suas relações, em seus vínculos, dispondo das coisas, tendo em vista uma determinada finalidade; conduzir condutas, articulando e incitando um conjunto de ações sobre ações possíveis, a partir de uma lógica econômica.

Por este motivo, Foucault sugere que talvez não tivesse escolhido o nome Securité, territoire, population para seu curso, na medida em que seu objetivo está na exumação de uma história da governamentalidade: "Par 'gouvernementalité, j'entends l'ensemble constitué par les institutions, les prócedures, analyses et réflexions, les calculs et les tactiques qui permetent d'exercer cette forme bien éspecifique, quoique très complexe, de pouvoir" ${ }^{16}$, que tem por alvo principal a população, por forma de saber a economia política, e por instrumento técnico fundamental os dispositivos de segurança.

A arte de governar, que se insinua desde o século XVI, expressa uma leitura anti-maquiavélica, que se opõe à posição transcendente do poder do príncipe, esboçando a transformação nos modos de exercer o poder em direção a um governo dos homens e das coisas, a partir do modelo econômico. E essa governamentalização (gouvernementalisation) do Estado, que foi fundamental na história do Ocidente, produziu-se a partir de três grandes pontos de apoio: a pastoral cristã, as novas técnicas diplomático-militares, e o surgimento da polícia (no sentido empregado ao termo nos séculos XVII e XVIII) - genealogias que aqui não teremos tempo de resgatar, mas que foram imprescindíveis enquanto movimento através do qual se constituía um campo de verdade com novos objetos de saber.

Especialmente em relação ao pastorado, esboçam-se em suas práticas modos específicos de individualização que, conforme Foucault, dão lugar, no cristianismo, a toda uma rede institucional que tem por escopo conduzir condutas, controlar os homens, revelando as formas incipientes de uma razão governamental, ou de uma arte de governar - e é a partir de tais práticas que devemos procurar a origem, o ponto de emergência e formação dessa governamentalidade que entra em cena no

${ }^{16}$ Ibidem, pg. 111.

Opinião Filosófica - ISSN: 2178-1176 - Editora Fundação Fênix. www.fundarfenix.com.br 
século XVI, definindo os limiares do Estado moderno. De modo geral, toda a história desses procedimentos de individualização humana no Ocidente está envolvida na história do pastorado, que, conforme Foucault, poderia ser entendida enquanto uma "história do sujeito" (histoire du sujet) ${ }^{17}$. Trata-se de um prelúdio a governamentalidade, através da constituição específica de um sujeito, subjetivado pela extração de uma verdade que lhe é imposta ${ }^{18}$.

Na medida em que os dispositivos de segurança levam em conta a dimensão da aleatoriedade, as relações de poder, diferentemente das relações de soberania, supõem o exercício da liberdade. Assim, a dinâmica entre esses três elementos - a ação de governar, o exercício da liberdade e a produção da verdade - produz uma característica reflexa, designando uma dimensão ética inafastável, na medida em que o governo dos outros relaciona-se com as formas do governo de si mesmo. De acordo com Foucault, essa reflexão sobre a noção de governamentalidade não poderia deixar de passar pelo âmbito de um sujeito que seria definido pela relação de si para consigo"19. Governar não se restringe, portanto, apenas ao governo dos outros, mas também pela relação que o sujeito estabelece consigo mesmo, produzindo-se na forma da verdade, como nas práticas da confissão ${ }^{20}$.

Agora, levando-se em consideração a quadra histórica atual, da qual emergem novas maneiras de ser e estar presente no mundo através da virtualidade das relações no espaço digital das redes sociais, não estaríamos diante de questões semelhantes, ainda que num contexto inteiramente novo? Não seriam os dispositivos algorítmicos o codinome contemporâneo dos dispositivos de segurança, produzindo efeitos de saber desde técnicas sofisticadas de armazenamento e análise de dados, até a otimização das interações entre os indivíduos e o seu ambiente?

Conforme veremos a partir de agora, o conceito de governamentalidade, examinado brevemente até aqui, mostra-se fundamental para a compreensão dos processos de subjetivação que emergem na contemporaneidade, no contexto da formação dos dispositivos algorítmicos. Na medida em que as interações humanas desenrolam-se sobre o terreno virtual das redes sociais - estruturadas sob uma

\footnotetext{
17 Ibidem, p. 187.

${ }^{18}$ Ibidem, p. 187.

${ }^{19}$ FOUCAULT, Michel. L’herméneutique du sujet. Cours au Collège de France (1981-1982). Paris: Seuil/Gallimard, 2001.

${ }_{20}$ FOUCAULT, Michel. Histoire de la sexualité, IV: les aveux de la chair. Paris: Gallimard, 2018.
} 
lógica algorítmica -, torna-se imprescindível uma análise que leve em conta a microfísica dessas relações, enquanto conjunto de mecanismos e de procedimentos, de modo a colocar em questão tanto suas táticas de poder, quanto seus efeitos de saber sobre o indivíduo, em sua percepção sobre si mesmo e o mundo.

\section{Governamentalidade algorítmica}

Parece-nos oportuno, diante disso, retomar o instrumental teórico foucaultiano, examinando a dinâmica das relações que emergem desde o espaço virtual, a fim de compreender o modo pelo qual os dispositivos algorítmicos produziram novos objetos de saber, bem como introduziram, a partir daí, uma nova maneira de governar condutas. Nesse sentido, Antoinette Rouvroy e Thomas Berns utilizam-se do conceito de governamentalidade algorítmica (gouvernementalité algorithmique), para dar conta do funcionamento dessas novas estruturas de poder-saber, decompondo-o em três elementos fundamentais: dataveillance, datamining e profiling.

Em primeiro lugar, as novas oportunidades de agregação e análise de dados (os big data) possibilitaram apreender a realidade social de maneira direta e imanente, diante de uma coleta massiva e automática de dados que não tem, necessariamente, um objetivo prévio, senão sua conservação bruta e dispersa, desvinculada de toda e qualquer subjetividade ou intencionalidade - o chamado dataveillance (vigilância de dados)

Ces données apparaissent ainsi constitutives d'un comportementalisme numérique généralise dès lors qu'elles expriment ni plus ni moins que les multiples facettes du réel, le dédoubant dans sa totalité, mais de manière parfaitement segmentée, sans faire sens collectivement, sinon comme dédoublement du réel ${ }^{21}$.

A novidade dessa coleta de dados está ligada ao fato de que cada elemento é reconduzido a sua natureza bruta, abstraído do contexto no qual apareceu. Quer estejamos tratando de uma compra na internet, de uma curtida numa publicação, de uma palavra utilizada, tais elementos são reduzidos a mero “dado”, desvinculado de significação própria. Por essa razão, “elles sont bien moins intrusives qu'une

${ }^{21}$ BERNS, Thomas; ROUVROY, Antoinette. Gouvernementalité algorithmique et perspectives d'émancipation. In: Réseaux, $\mathrm{n}^{0} 1,2013$, p. 169. 
carte de fidélité, et elles semblent ne pas mentir, c'est-à-dire pouvoir être considérées comme parfaitement objectives” ${ }^{22}$, constituindo, deste modo, uma evitação da subjetividade.

O segundo momento da governamentalidade algorítmica se dá a partir do datamining (mineração/tratamento de dados), no qual essas quantidades massivas de dados são "tratadas", estabelecendo sutis correlações entre eles. Conforme Rouvroy e Berns, parece fundamental notar que "nous nous trouvons ainsi face à une production de savoir (des savoirs statistiques constitués de simples corrélations) à partir d'informations non triées, et donc parfaitement hétérogènes” ${ }^{23}$, que, estando automatizadas, demandam um mínimo de intervenção humana e dispensam toda forma

de hipótese prévia.

Le propre de ce qu'on appelle le machine learning est somme toute de rendre directement possible la production d'hypothèse à partir des données elles-mêmes. De la sorte, nous nous trouvons à nouveau face à l'idée d'un savoir dont l'objectivité' pourrait paraitre absolue, puisqu'il serait éloigné de toute intervention subjective [...] Les normes semblent émerger directement du réel lui-même. ${ }^{24}$

Os dispositivos algorítmicos intensificam e sofisticam os mecanismos dos dispositivos de segurança, examinados anteriormente, extraindo um efeito de saber da relação estabelecida entre os dados coletados. Porém, essas correlações não se dão desde uma hipótese - como, por exemplo, no caso das estatísticas, quando se quer estabelecer uma relação entre a mortalidade de uma determinada população e o seu índice de desenvolvimento humano -, mas emergem desde o próprio real, de maneira imanente e direta.

Assim, o terceiro momento da governamentalidade algorítmica é descrito pela elaboração de perfis (profilage ${ }^{25}$, ou perfilização, numa tradução aproximada para o português) a partir dos dados coletados e organizados. Essa perfilização leva em conta uma dicotomia, apontada por Van Otterlo, entre os dados coletados ao nível individual, bem como aqueles correspondentes a um nível agregado: no primeiro caso, trata-se de dados consistentes de peças de informação, observáveis como tais, até mesmo pelo indivíduo com o qual elas se relacionam, enquanto no

\footnotetext{
${ }^{22}$ Ibidem, p. 170.

23 Ibidem, p. 170.

24 Ibidem, p. 170.

25 Ibidem, p. 166. 
segundo caso, as correlações estabelecidas entre os dados de um determinado indivíduo não podem ser por ele observadas ou imaginadas, sendo antes a ele aplicadas, com o objetivo de inferir deles um saber, bem como previsões e probabilidades comportamentais. "The crucial difference is that information at the individual level is actually observed, that is, it is factual knowledge. Knowledge at the profiling level is not usually available to the individual user and is often not observed. Instead the profiling is applied to the individual user to infer additional facts, preferences or assumed intentions [...]"26.

A governamentalidade algorítmica opera, portanto, uma ação por antecipação aos comportamentos individuais, a partir da elaboração de perfis (ou, como optamos chamar aqui, perfilização), a fim de "éviter l"imprévisible, de faire en sorte que chacun soit véritablement lui-même” ${ }^{27}$. De maneira mais precisa, Rouvroy e Berns definem a governamentalidade algorítmica da seguinte forma:

\begin{abstract}
Par gouvernementalité algorithmique, nous désignons dès lors globalement un certain type de rationalité (a)normative ou (a)politique reposant sur la récolte, l'agrégation et l'analyse automatisée de données en quantité massive de manière à modéliser, anticiper et affecter par avance les comportements possibles. Si on se réfère au socle général de la pensée statistique, les déplacements apparents qui seraient produits actuellement par le passage du gouvernement statistique au gouvernement algorithmique, et qui donneraient sens à un phénomène de raréfaction des processus de subjectivation, sont donc les suivants: tout d'abord, une apparente individualisation de la statistique (avec l'antinomie évidente qui s'exprime ainsi), laquelle ne transiterait plus (ou ne semblerait plus transiter) par des références à l'homme moyen, pour donner place à l'idée qu'on deviendrait à soi-même son propre profil automatiquement attribué et évolutif en temps réel. Ensuite, un souci accru d'éviter le danger d'une pratique statistique tyrannique qui réduirait l'objet statistique à du bétail, en veillant à ce que cette pratique statistique se développe comme si notre accord était donné, puisque c'est en tant que nous sommes chacun uniques que le mode de gouvernement par les algorithmes prétend s'adresser à chacun à travers son profil ${ }^{28}$
\end{abstract}

A governamentalidade algorítmica produz uma normatividade aparentemente “democrática”, na medida em que põe a funcionar uma relação nãoseletiva com o mundo, descolada de qualquer subjetividade ou intencionalidade na coleta de dados e elaboração de perfis. Sobretudo, essas práticas estatísticas

\footnotetext{
${ }^{26}$ VAN OTTERLO, Martjin. A machine learning view on profiling. In: Privacy, due process and the computational turn - Philosophers of law meet philosophers of techonology. Abingdon: Routledge, 2013, p. 44.

${ }_{27}$ BERNS, Thomas; ROUVROY, Antoinette. Gouvernementalité algorithmique et perspectives d'émancipation. In: Réseaux, $\mathrm{n}^{0} 1,2013$, p. 173.

28 Ibidem.
} 
contemporâneas, na forma dos dispositivos algorítmicos, incluem nelas mesmas a expressão de uma adesão tácita dos indivíduos ao seu funcionamento. Daí que, conforme os autores, estarmos diante de um declínio da reflexividade subjetivante, na medida em que a produção de saber, através do datamining, distancia-se de qualquer contestação por aqueles nos quais se apoia.

Segundo Rouvroy e Berns,

La gouvernementalité algorithmique ne produit aucune subjectivation, elle contourne et évite les sujets humains réflexifs, elle se nourrit de donnéesinfra-individuelles insignifiantes en elles-mêmes, pour façonner des modèles de comportements ou profils supra-individuels sans jamais en appeler au sujet, sans jamais l'appeler à rendre compte par lui-même de ce qu'il est ni de ce qu'il pourrait devenir. Le moment de réflexivité, de critique, de récalcitrance, nécessaire pour qu'il y ait subjectivation semble sans cesse se compliquer ou être postposé ${ }^{29}$.

Nesse sentido, a concepção de uma governamentalidade algorítmica introduz a ideia de práticas a-normativas, ou a-subjetivantes, na medida em que ela evita a subjetividade dos indivíduos no funcionamento de seus mecanismos. Não estamos, portanto, diante do risco de extinção do indivíduo, ou de sua dessubjetivação, mas, ao contrário, diante do fenômeno de rarefação dos processos e ocasiões de subjetivação, de dificuldade em tornar-se sujeito ${ }^{30}$. Pode-se dizer, desta forma, que a governamentalidade algorítmica nos expulsa da perspectiva genealógica, visto que não é a questão da antecipação dos comportamentos que deveria surpreender-nos, senão o fato de que consideremos o paradoxo "selon lequel, désormais, pour éradiquer ou minimiser l'incertitude, on s'en remet à des 'appareils' non-intentionnels, c'est-à-dire à des machines a-signifiantes, en abandonnant de la sorte l'ambition de donner de la signification aux événements, qui ne sont d'ailleurs plus nécessairement traités comme des événements [...]"31, diante da possibilidade de serem decompostos e reagregados a partir de conjuntos de dados.

Assim, conforme Rouvroy e Berns,

Le sujet de la gouvernementalité algorithmique est, de plus en plus, saisi par le " pouvoir ", non pas à travers son corps physique, ni à travers sa conscience morale - prises traditionnelles du pouvoir dans sa forme juridico-discursive mais à travers les multiples " profils » qui lui sont

\footnotetext{
29 Ibidem, p. 173.

30 Ibidem, p. 180.

${ }^{31}$ Ibidem, p. 174 . 
assignés, souvent de manière automatique sur la base des traces numérisées de son existence et de ses trajectoires quotidiennes. La gouvernementalité algorithmique correspond assez bien à ce que Foucault visait déjà sous son concept de dispositif de sécurité32.

A ação dos dispositivos algorítmicos se dá sobre o ambiente, de modo a organizar as possibilidades do indivíduo desde a rede de correlações estabelecidas por suas interações no meio digital. Estabelecem-se entre os elementos heterogêneos que compõem a rede social, por exemplo, toda uma malha de relações cambiantes, sob o imperativo de interatividade - que consolida a digitalização da vida social, através da qual os sistemas informáticos passam a se encarregar das decisões e possibilidades da vida cotidiana (SADIN, 2015). O problema colocado em questão pelos autores, portanto, não está ligado a processos de “individuação", mas, justamente, na indiferença desses dispositivos algorítmicos em relação ao indivíduo, na medida em que seus objetos de saber, bem como seus objetivos de intervenção, são a dupla estatística de nossa individualidade, ou seja, as relações estabelecidas pelos, e a partir de, nossos perfis. Conforme Pablo Rodríguez, os indivíduos já não poderiam ser considerados, desta forma, "individuais" no reino da informação, "pero lo que los hace individuos al término del processo será el resultado de alguna suerte de 'dividuación': transformación de indivíduos en datos y recomposición posterior que da como resultado otro individuo relacionado, pero en principio no igual al individuo antes de ser transformado" 33 .

Deste modo, a questão não é ser privado de nossas informações, ou sermos obrigados a cedê-las, senão decorre, fundamentalmente, "du fait que notre double statistique est trop détaché de nous, que nous n'avons pas de 'rapport' avec lui, alors même que les actions normatives contemporaines se suffisent de ce double statistique pour être efficaces." 34.

Dito de outro modo,

[...] là où le confessionnal fabrique le sujet de l'introspection qui sonde son âme, sa vertu, ses désirs et ses intentions les plus profondes [...] là où la loi produit des sujets de droit soucieux de leur égalité et de l'impartialité des procédures, là où l'homme moyen apparaissait comme trop moyen par rapport à tout sujet singulier susceptible de se constituer contre cette moyenne, le gouvernement algorithmique ne donne ni lieu, ni prise à

${ }^{32}$ Ibidem, p. 174-175.

33 RODRÍGUEZ, Pablo. Las palabras en las cosas. Poder, saber y subjetivación, entre algoritmos y biomoléculas. $1^{\text {a }}$ ed. Ciudad Autónoma de Buenos Aires: Cactus, 2019, p. 453.

34 BERNS, Thomas; ROUVROY, Antoinette. Gouvernementalité algorithmique et perspectives d'émancipation. In: Réseaux, ${ }^{0} 1,2013$, p. 181. 
aucun sujet statistique actif, consistant, réflexif susceptible de le légitimer ou de lui résister 35

São as relações, os dados transmitidos, as interações interindividuais, portanto, o objeto do governo algorítmico, em proveito dos quais os sujeitos seriam, deste modo, evitados, ou ao menos impedidos, em seus processos de subjetivação: "Les données transmises sont des relations et ne subsistent que comme relations; les connaissances générées sont des relations de relations; et les actions normatives qui en découlent sont des actions sur des relations (ou des environnements) référées à des relations de relations." ${ }^{36}$. Em suma, um governo de relações, que em suas práticas procura organizar o possível, e cujo campo de ação não está situado no presente, mas no futuro dos acontecimentos, através da configuração de um acaso sistematizado.

Nesse sentido, conforme Frank Pasquale,

Search engines host billions of queries per day. They "answer" more and more of them without the asker ever having to click through to another site. They keep track of our friends, real and virtual. They find our entertainment. They rank and rate everything for us, from movies to doctors to hotels. [...] These new masters of media are more than just conveniences. Thanks both to their competence and our inertia, they often determine what possibilities reach our awareness at all. They are guides; they influence, sometimes quite profundly, our decisions about what we do and think and buy (and what we don't). They are revolutionaries ${ }^{37}$.

Conforme Antoinette Rouvroy, o regime de otimização algorítmica não é, portanto, um "regime de verdade", que obrigue os sujeitos a falar ao modo de uma confissão, mas, antes, um regime de indistinção entre os sinais numéricos, aos quais os dados coletados são reduzidos - a-significantes, porém calculáveis - e o mundo ${ }^{38}$. Sinais que transpiram da trajetória de relações e interações dos indivíduos, de modo a constituir perfis de dados correlacionados, duplicatas da individualidade, a partir das quais se infere um determinado saber, bem como, em igual medida, um conjunto de ações sobre ações possíveis.

\footnotetext{
35 Ibidem, p. 181.

${ }^{36}$ Ibidem, p. 184.

37 PASQUALE, Frank. The black box society: the secret algorithms that control money and information. Harvard University Press, 2015, p. 59.

${ }^{38}$ ROUVROY, Antoinette. Gouverner hors les normes: la gouvernementalité algorithmique. In: Lacan quotidien, v. 733, 2017.
} 
De modo que, conforme a autora, na dimensão virtual dos dispositivos algorítmicos já não estamos separados do mundo, senão com ele formamos uma unicidade, sendo digitalmente um único corpo numérico, junto a um mundo igualmente numérico, imersos e atravessados por fluxos ${ }^{39}$. Nesse regime de indistinção entre os sinais numéricos e as coisas, desqualifica-se, então, aquilo que, de acordo com Rouvroy, constitui o único espaço incalculável e imprevisível sobre uma pessoa: a maneira sempre singular de como ela dá conta de si mesma e para si mesma, de como ela se percebe em seus próprios gestos e ações.

L'optimisation algorithmique des interactions entre l'individu et son milieu passe pour une rationalisation des formes à travers lesquelles nous (nous) gouvernons, auxquelles elle substitue des profils (corrélations entre données), éminemment évolutifs, extrêmement plastiques, précédant les individus das leus comportements, trajectoires, choix - des moules n'ayant plus rien de la fixité des traces, se comportant plutôt comme des empreintes qui précéderaient nos pas ou comme des ombres jamais tout à fait adéquates qui auraient, sur les mouvements de leurs proies, toujours une longueur d'avance40.

A governamentalidade algorítmica não descreve, portanto, um regime de normalização, mas um sistema imunitário da realidade numérica, operando contra toda e qualquer heterogeneidade incalculável, de maneira a neutralizar as subjetividades e dispensar o mundo e suas representações, em nome de uma realidade feita de fluxos numéricos - de modo a retirar dos sujeitos sua capacidade de decidir, bem como de projetar coletivamente suas possibilidades.

\section{Considerações finais}

Essa aplicação, ao nível dos comportamentos individuais, da norma que se extrai do meio digital - sob a aparência de personalização das ofertas de informação, serviços e produtos -, leva a uma colonização do espaço público por uma esfera privada hipertrofiada, cuja dinâmica, segundo Rouvroy e Berns, deveríamos investigar, "au point de faire craindre que les nouveaux modes de filtrage de l'information aboutissent à des formes d'immunisation informationnelles

\footnotetext{
39 Ibidem.
}

40 Ibidem. 
favorables à une radicalisation des opinions et à la disparition de l'expérience commune ${ }^{41}$.

Diante disso, como manter aberta a possibilidade de um projeto político, e a sobrevivência dos sujeitos? Como fazer de sorte que, conforme Rouvroy, os "animais heterocrônicos" (animaux hétérochroniques) que somos não sejam levados em conta apenas enquanto agregados temporários, enquanto conjunto de dados na forma de perfis? Como, em face dessa espécie de "hiperindividualismo otimizador" (l'hyper individualisme optmisateur), encontrar maneiras de revitalizar o espaço público, o "comum", enquanto espaço de deliberação e projeção de possibilidades, ou seja, enquanto lugar de abertura a novas possibilidades políticas?

\section{Referências}

BERNS, Thomas; ROUVROY, Antoinette. Gouvernementalité algorithmique et perspectives d'émancipation. In: Réseaux, $\mathrm{n}^{0}$ 1, p. 163-196, 2013.

FOUCAULT, Michel. Histoire de la sexualité, I: la volonté de savoir. Paris: Gallimard, 1976.

FOUCAULT, Michel. L'herméneutique du sujet. Cours au Collège de France (19811982). Paris: Seuil/Gallimard, 2001.

FOUCAULT, Michel. Securité, territoire, population. Cours au Collège de France (1977-1978). Paris: Seuil/Gallimard, 2004.

FOUCAULT, Michel. Histoire de la sexualité, IV: les aveux de la chair. Paris: Gallimard, 2018.

PASQUALE, Frank. The black box society: the secret algorithms that control money and information. Harvard University Press, 2015.

RODRÍGUEZ, Pablo. Las palabras en las cosas. Saber, poder y subjetivación entre algoritmos y biomoléculas. $1^{\mathrm{a}}$ ed. Ciudad Autónoma de Buenos Aires: Cactus, 2019.

ROUVROY, Antoinette. Gouverner hors les normes: la gouvernementalité algorithmique. In: Lacan Quotidien, v. 733, 2017.

SADIN, Eric. La vie algorithmique. Crítique de la raison numérique. Paris: Éditions L’Échappee, 2015.

${ }^{41}$ BERNS, Thomas; ROUVROY, Antoinette. Gouvernementalité algorithmique et perspectives d'émancipation. In: Réseaux, $\mathrm{n}^{\mathrm{0}} 1,2013, \mathrm{p} .167$. 
VAN OTTERLO, Martjin. A machine learning view on profiling. In: Privacy, due process and the computational turn - Philosophers of law meet philosophers of techonology. Abingdon: Routledge, p. 41-64, 2013.

Recebido em: 15/07/2021. Aprovado em: 23/07/2021. Publicado em: 05/08/2021. 